\title{
Validation of smoking cessation self-reported by patients with chronic obstructive pulmonary disease
}

This article was published in the following Dove Press journal:

International Journal of General Medicine

21 January 20II

Number of times this article has been viewed

\author{
Sander R Hilberink' \\ Johanna E Jacobs' \\ Sanne van Opstal ${ }^{2}$ \\ Trudy van der Weijden ${ }^{2}$ \\ Janine Keegstra' \\ Pascal LJ Kempers ${ }^{3}$ \\ Jean WM Muris ${ }^{2}$ \\ Richard PTM Grol' \\ Hein de Vries ${ }^{4}$ \\ 'IQ Healthcare, Radboud University \\ Nijmegen Medical Centre, Nijmegen, \\ The Netherlands; ${ }^{2}$ Department \\ of General Practice, Maastricht \\ University, Research Institute \\ CAPHRI, Maastricht, The Netherlands; \\ ${ }^{3}$ Department of Health Risk, Analysis \\ and Toxicology, ${ }^{4}$ Department of \\ Health Promotion and Health, \\ Maastricht University, Maastricht, \\ The Netherlands
}

Purpose: The present study reports on the biochemical validation of the self-reported smoking status of patients with chronic obstructive pulmonary disease (COPD). The objective is to establish the proportion of overestimation of self-reported success rates.

Methods: A cross-sectional smoking-status validation study including 60 patients with COPD who reported that they had stopped smoking. In the analysis of urine samples, a cut-off point of $50 \mathrm{ng} / \mathrm{mL}$ of cotinine was used.

Results: At the time of biochemical validation, 55 patients reported that they had quit smoking while five patients resumed smoking. Smoking status was biochemically confirmed for 43 patients (78\%) and 12 patients (22\%) were classified as smokers. The sensitivity of the self-report of smoking was $29 \%$ and the specificity was $100 \%$.

Conclusion: Many primary care patients with COPD do not provide valid information on their smoking status, which hamper adequate therapeutic interventions. Integration of biochemical validation in daily care could overcome this problem, but may harm the doctor-patient relationship.

Keywords: chronic obstructive pulmonary disease, smoking cessation, biochemical validation, general practice, outcome measurement

\section{Introduction}

Smoking cessation interventions provided in general practice have proven to be effective. ${ }^{1,2}$ The role of general practitioners (GPs) in supporting their patients who want to quit smoking is documented in international guidelines. ${ }^{3}$ Addressing the smoking status of patients can be difficult, but GPs are more likely to do so if their patient suffers from smoking-related complaints. ${ }^{4}$ The GPs' advice and support encourages patients to quit smoking. How do physicians learn whether their support was successful? Are they able to rely on the patients' self-reports?

Chronic obstructive pulmonary disease (COPD) is illustrative of a smoking-related disease. This slowly progressive disease is characterized by airflow obstruction with related symptoms such as cough, sputum production, dyspnea, and wheeze and it is mainly caused by smoking. ${ }^{5-7}$ Among patients with COPD, smoking prevalence varies from $38 \%-52 \%$ in seven countries. ${ }^{8}$ Because smoking accounts for $80 \%-90 \%$ of COPD, one may assume that many patients find it difficult to quit smoking. Smoking cessation slows down the rate of deterioration of lung function, improves COPD prognosis, and may prolong life expectancy for these patients. ${ }^{9-12}$ Consequently, smoking cessation is the most important element of therapy for COPD, ${ }^{5}$ and it is necessary to measure the results of smoking cessation interventions during the therapeutic process.
Correspondence: Sander R Hilberink IQ Healthcare, PO Box 910I, IQ II4, 6500 HB Nijmegen, The Netherlands

$\mathrm{Tel}+3 \mathrm{I} 243615305$

Fax +3I 243540166

Email s.r.hilberink@hetnet.nl 
The self-report of smoking cessation in community surveys among the general population is reasonably valid. ${ }^{13-16}$ For smokers specially at risk (eg, coronary disease, COPD, pregnancy), the self-reported smoking status is less reliable. ${ }^{13,17-20}$ Biochemical testing is recommended for specific populations where quitting is very desirable. ${ }^{21}$ Cotinine, the major proximate metabolite of nicotine, has been widely used as a biomarker of tobacco exposure. The relatively long half-life of cotinine (16-20 hours) enables detection for a few days after cessation of tobacco use. The sensitivity and specificity of cotinine are high, 97\% and $99 \%-100 \%$, respectively, ${ }^{22,23}$ and it is therefore the preferred substance to measure.

Although the rationale for biochemical validation of the smoking status of patients with increased health risks is evident, the Lung Health Study found little discrepancy between self-reports and cotinine measurement of patients with early-staged COPD. ${ }^{24}$ Nevertheless, the patients who had received smoking cessation support misreported their smoking status more often than patients who had not ( $6 \%$ versus $1 \%)$. This may suggest that participation in a smoking cessation program increases the social desirability bias. Participation of COPD patients in an intensive smoking cessation program had even higher rates $(52 \%)$ of misreporting after biochemical testing of their smoking status. ${ }^{20}$ Smoking cessation interventions, as carried out in general practice during routine follow-up, are generally less intensive than these programs. ${ }^{20,24}$ The question is, then, how accurate are the self-reports of COPD patients if they have been subjected to a less intensive smoking cessation intervention in the patients' own general practice? As smoking cessation support is recommended in routine general practice for patients with COPD, it is important for GPs to obtain valid information about their patients' smoking status. This is necessary to use smoking cessation programs effectively and to adjust the medical therapy to their results. Since the literature is inconclusive about the extent of misreporting of the smoking status of COPD smokers in general practice, the present study examines the validity of self-reported smoking status of COPD patients participating in a study testing a moderately intensive smoking cessation program in daily general practice.

\section{Methods and materials}

The present study is a validation study of self-reported smoking status assessed by urine cotinine analysis. The data were collected from all patients who reported that they had not smoked in the past 7 days.

\section{Subjects and procedure}

A software program using Anatomical Therapeutical Chemical (ATC) prescription codes and International Classification of Primary Care (ICPC) diagnosis codes selected potential patients with COPD from general practices. The criteria included age of 35 years or more and a diagnosis recorded as COPD, or as ICPC code R95/96, or at least three prescriptions of bronchodilators (ATC code R03a/bc), and/or at least two prescriptions of inhaled anti-inflammatory medication in the last year (ATC code R03). The GPs had to confirm the diagnosis of the selected patients. Patients were eligible to participate if they were: 1) currently smoking; 2) suffering from COPD according to the GP's diagnosis; 3) not being treated by a chest physician; 4) in command of the Dutch language; and 5) not suffering from any serious physical or psychological co-morbidity. The participants consented to participate in an intervention study in general practice and were allocated to one of the three study groups (including a control group). The patient-directed intervention tailored to general practice patients with COPD was based on the minimal intervention strategy. ${ }^{2}$ GPs applied a stagebased protocol, providing information, smoking cessation counseling, and advised the use of pharmacological cessation aids (ie, nicotine replacement therapy or bupropion-SR). The patient education tools consisted of a leaflet especially developed for COPD smokers and a videotape. The patients included in the present study self-reported to have quit smoking during the 12-month follow-up. More information about patient inclusion and the smoking cessation program can be found elsewhere. ${ }^{25-27}$ Figure 1 shows that of the 667 enrolled patients, 86 patients said at the 12-month follow-up that they were not smoking: however, 22 patients (26\%) did not enter the present study (17 patients refused and five patients were too ill), three patients were excluded because of their current nicotine replacement therapy, and one patient was excluded due to missing data. Hence, our sample consisted of 60 patients. The procedures followed were approved by the Committee on Human Experimentation, Maastricht University, The Netherlands.

\section{Measurement}

The participants were briefed at the beginning of the study (1 year prior to the assessment of their smoking status) about possible self-report crosschecks with biochemical validation. They received a baseline questionnaire $(\mathrm{Q} 0)$ to complete at home to provide the baseline characteristics of the sample including their motivation to quit smoking, ${ }^{28}$ severity of nicotine dependence (Fagerström Test of Nicotine 


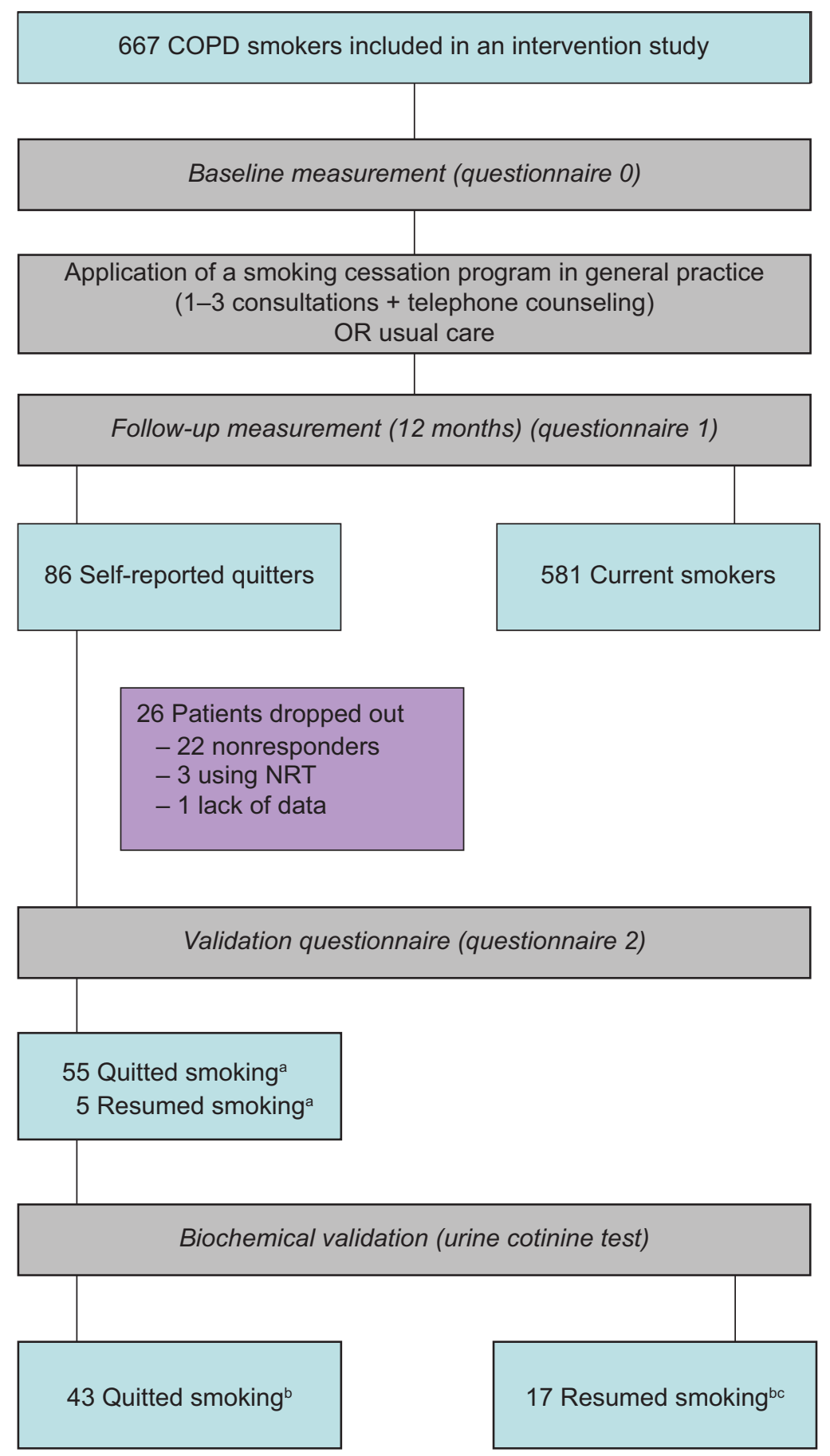

Figure I Flow of the biochemical validation study.

Notes: aSelf-report, 'Biochemically verified, Including the 5 self-reported resumed smokers.

Dependence), ${ }^{29}$ and COPD symptoms (Medical Research Council). ${ }^{30}$ Twelve months later, they received another questionnaire (Q1) regarding their smoking status (7-day point prevalence). The patients who reported that they had quit smoking were included in the validation study and received a letter in which they were asked to visit the general practice within a week to produce and hand over a urine sample to be sent to the researchers for analysis. If they were unable to visit the practice, a research assistant collected the sample during a home visit. Furthermore, they were asked to complete an additional short questionnaire at the exact time of the sample production (Q2), which included their current smoking behavior and use of nicotine substitution. All questionnaires were self-administered and were used for 
research only. The results of the biochemical validation of the self-reported smoking status were not communicated to the patients' GPs.

The amount of urinary cotinine was measured by radioimmunoassay. Radioimmunoassay involves the use of antibodies to cotinine. The double-antibody technique was used to separate free-labeled antigen from antibody-bound antigen. A reaction mixture containing $0.1 \mathrm{~mL} 3 \mathrm{H}$-cotinine, appropriately diluted antiserum and buffer $(0.14 \mathrm{M} \mathrm{NaCl}$, $0.01 \mathrm{M}$ Tris- $\mathrm{HCl}$, and $0.1 \%$ gelatin) was incubated at $37^{\circ} \mathrm{C}$. For inhibition experiments, $0.1 \mathrm{~mL}$-aliquots containing known amounts of standard or dilution of the urine-samples were added in place of buffer. Goat anti-rabbit immunoglobulin was added and the mixture was incubated at $4{ }^{\circ} \mathrm{C}$ overnight. To control for nonspecific binding, normal rabbit serum was used in place of immune serum. The precipitate was collected by centrifugation at $4200 \mathrm{rpm}$ for 45 minutes at $4^{\circ} \mathrm{C}$, the supernatant decanted, and the walls of the tubes wiped dry. To count the $3 \mathrm{H}$-cotinine, the precipitate was dissolved in $0.1 \mathrm{~mL} 0.1 \mathrm{~N}$ sodium hydroxide before adding $2.5 \mathrm{~mL}$ scintillation fluid. Eventually the rate of radioactivity was counted and the amount of cotinine could be calculated from the linear portion of the standard curve. The results were reported in nanograms per milliliter.

\section{Analyses}

The cut-off point for both smokers and nonsmokers was $50 \mathrm{ng} / \mathrm{mL}^{21}$ We used the contingency coefficient (twotailed test) to analyze the association of the self-reported status and the biochemically confirmed smoking status. Moreover, the sensitivity and specificity of the self-report were calculated. Differences in cotinine levels were analyzed by means of the Mann-Whitney $U$ test (two-tailed). The data were analyzed with SPSS software (v. 14.0; SPSS Inc., Chicago, IL).

\section{Results}

Table 1 shows the baseline characteristics (Q0) of the 60 participants. Seven patients were allocated to the control group. The sample included 59\% men, and the participants had a mean age of almost 60 years. At baseline, 38\% indicated they wanted to quit smoking within 1 month, 34\% wanted to quit within 6 months, and $28 \%$ were not motivated to quit smoking. Thirty percent suffered from chronic sputum production and $35 \%$ from chronic coughing.

Forty-three patients $(72 \%)$ of the 60 patients were classified by the urine cotinine test as nonsmokers (median cotinine level $13.0 \mathrm{ng} / \mathrm{mL}$, quartile range 6.0-22.0), and
Table I Characteristics of 60 self-reported quitters

\begin{tabular}{ll}
\hline Variable & Sample \\
\hline Men (\%) & 59 \\
Mean age (SD) & $59.9($ II I.0) \\
Stages of change (\%) & \\
$\quad$ Preparer & 38 \\
$\quad$ Contemplator & 34 \\
Precontemplator & 28 \\
Mean number of cigarettes per day (SD) & $16.4(8.2)$ \\
Mean FTND (SD) & $4.2(2.4)$ \\
Chronic sputum (\%) & 30 \\
Chronic coughing (\%) & 35 \\
Mean dyspnea, range 0-3 (SD) & $1.3(1.1)$ \\
\hline
\end{tabular}

Note: Percentages are rounded.

Abbreviations: FTND, Fagerström Test of Nicotine Dependence; SD, standard deviation.

17 patients $(28 \%)$ were classified as smokers (median cotinine level $134.0 \mathrm{ng} / \mathrm{mL}$, quartile range 68.0-194.0) (z-6.00; $P<0.001)$.

During the time of the biochemical validation, 12 of the 17 patients, who were classified as smokers, reported that they did not smoke (11 had taken part in the smoking cessation intervention) and five said that they had smoked recently (three had attended the intervention). Thus, the selfreporting of $43(78 \%)$ of the 55 patients who claimed that they were not smoking at the time of taking the urine sample (Q2) corresponded with the classification by the cotinine level and 12 (22\%) patients did not correspond (see Table 2) (contingency coefficient $0.43 ; P<0.001$ ). The self-report had $29 \%$ sensitivity for detecting actual smoking, and a specificity of $100 \%$.

\section{Discussion}

We found a misreport rate of $22 \%$. For COPD patients both lower ${ }^{24}$ and higher ${ }^{20}$ misreport rates have been described. It is important to bear in mind that the self-reporting of smoking status was combined with providing a urine sample for biochemical validation. This combination might result in a lower misreport rate, because the patients are aware that their self-reports will be checked. If this is true, then the misreporting of $22 \%$ could be an underestimation. If the patients who refused to be tested $(n=22)$ were still smoking, then the misreport rate could be as high as $42 \%$. The real misreporting

Table 2 Association between self-reported quit rates and biochemically validated quit rates

\begin{tabular}{lll}
\hline Biochemical validation & \multicolumn{2}{l}{ Self-report (questionnaire 2) } \\
\cline { 2 - 3 } & Not smoking & Smoking \\
\hline Not smoking & 43 & 0 \\
Smoking & 12 & 5 \\
\hline
\end{tabular}


figures will probably lie somewhere between these figures. The low sensitivity of self-reports (29\%), even in a situation where the report may be biochemically verified, shows their restricted value in clinical smoking cessation programs for high-risk populations.

There may be several explanations for the difference between self-reported and biochemically determined smoking status. First, the time elapsed between the two measurements (Q1 and Q2) might be a factor. One of the reasons for the great percentage of misreports might be that patients had relapsed to smoking after Q1. We tried to overcome this problem by reassessing the smoking status $(\mathrm{Q} 2)$, which showed that some of the COPD patients had indeed resumed smoking. Second, the results did not account for the influence of environmental tobacco smoke, which is known to influence cotinine levels. ${ }^{31}$ However, since our cut-off point was $50 \mathrm{ng} / \mathrm{mL}$ this is not likely. Third, misreporting might be triggered by the fact that patients participated in a smoking cessation intervention. ${ }^{24}$ Of the 12 patients who misreported their smoking status, 11 participated in the intervention. Interestingly, while Monninkhof et al report a misreport rate of $52 \%$ for COPD patients participating in an intensive smoking cessation intervention, they found a misreport rate of $13.6 \%$ at baseline (ie, for patients not participating in a smoking cessation intervention).$^{20}$ This suggests that although biochemical validation of self-reported smoking status of COPD patients is recommendable in any situation to obtain valid data, participation in an intensive smoking cessation program may increase these misreport rates.

Although the number of participating patients might limit the external validity of our results, our population is a reflection of the self-reported quitters with COPD from a large sample of patients treated in general practice. However, the small numbers of patients that misreported their smoking status hampered in-depth analyses of factors contributing to (in)valid self-reports.

Our results show that the self-reporting of quitting by COPD smokers is not always trustworthy. A general question remains how should medical professionals deal with patients who need ongoing medical attention for chronic smokingrelated diseases and who may lie about their smoking behavior? In our study we did not communicate the results of the biochemical validation to the GPs because they were used for research purposes, but some argue that biochemical validation should be integrated in the therapeutic process.

Clinical practice teaches that some physicians are reluctant to treat patients with COPD who persist in smoking. They argue that treating symptoms that are consciously caused by the patient's persistence in an unhealthy lifestyle is a waste of valuable time and effort. In this case, biochemical validation might be a good instrument for assessing smoking and for increasing the pressure on the patient to quit smoking. Apart from the questions of which measurement is feasible in routine daily general practice and which measurement has good sensitivity and specificity (eg, carbon monoxide or cotinine measures), this harsh approach might damage the relationship with the patient and it questions the ethical codes for health professionals. Misreporting one's smoking behavior, lying about it, social desirability bias; whatever we call it, it is probably a result of feeling ashamed or guilty, and therefore one might ask whether it is wise to apply biochemical validation tests as a sort of lie detector. Besides the ethical problem, Bize et al found in their systematic review no sufficient evidence that providing this kind of biomedical feedback contributes to successful smoking cessation. ${ }^{32}$ Therefore, in spite of the importance of getting a reliable view of the patients' smoking status in the context of therapeutic general practice interventions, we do not have a clear-cut answer on how best to obtain this information in a real-life general practice setting. Ethical issues concerning the patient's own responsibility and the effectiveness of biomedical feedback should be weighed against the professional need of getting reliable information about therapeutic patient outcomes.

\section{Acknowledgments}

This study was financed by the Dutch Asthma Foundation, Netherlands Organisation for Health Research and Development (ZonMW), Pharmacia, and GlaxoSmithKline.

\section{Disclosure}

The authors declared no conflicts of interest in this work.

\section{References}

1. Stead LF, Bergson G, Lancaster T. Physician advice for smoking cessation. Cochrane Database Syst Rev. 2008;2:CD000165.

2. Pieterse ME, Seydel ER, Vries H de, Mudde AN, Kok GJ. Effectiveness of a minimal contact smoking cessation program for Dutch general practitioners: a randomized controlled trial. Prev Med. 2001;32(2): 182-190.

3. Zwar NA, Richmond RL. Role of the general practitioner in smoking cessation. Drug Alcohol Rev. 2006;25(1):21-26.

4. Coleman T, Murphy E, Cheater F. Factors influencing discussion of smoking between general practitioners and patients who smoke: a qualitative study. Brit J Gen Pract. 2000;50(452):207-210.

5. Rabe KF, Hurd S, Anzueto A, et al. Global strategy for the diagnosis, management, and prevention of chronic obstructive pulmonary disease: GOLD Executive Summary. Am J Respir Crit Care Med. 2007;176(6): 532-555.

6. Mannino DM. COPD: Epidemiology, prevalence, morbidity and mortality, and disease heterogeneity. Chest. 2002;121(5 Suppl):S121-S126. 
7. Decramer M, Gosselink R, Bartsch P, et al. Effect of treatments on the progression of COPD: report of a workshop held in Leuven, 2004 March 11-12. Thorax. 2005;60(4):343-349.

8. Wouters EF. Economic analysis of the confronting COPD survey: an overview of results. Respir Med. 2003;97(Suppl C):S3-S14.

9. Scanlon PD, Connett JE, Waller LA, et al. Smoking cessation and lung function in mild-to-moderate chronic obstructive pulmonary disease. The Lung Health Study. Am J Respir Crit Care Med. 2000;161(2 Pt 1): 381-390.

10. Anthonisen NR, Connett JE, Kiley JP, et al. Effects of smoking intervention and the use of an inhaled anticholinergic bronchodilator on the rate of decline of $\mathrm{FEV}_{1}$. The Lung Health Study. JAMA. 1994; 272(19):1497-1505.

11. Willemse BWM, Postma DS, Timens W, ten Hacken NH. The impact of smoking cessation on respiratory symptoms, lung function, hyperresponsiveness and inflammation. Eur Respir J. 2004;23(3): 464-476.

12. Hersh CP, DeMeo DL, Al-Ansari E, et al. Predictors of survival in severe, early onset COPD. Chest. 2004;126(5):1443-1451.

13. Velicer WF, Prochaska JO, Rossi JS, Snow MG. Assessing outcome in smoking cessation studies. Psychol Bull. 1992;111(1):23-41.

14. Wagenknecht LE, Burke GL, Perkins LL, Haley NJ, Friedman GD. Misclassification of smoking status in the CARDIA study: A comparison of self-report with serum cotinine levels. Am J Public Health. 1992;82(1):33-36.

15. Patrick DL, Cheadle A, Thompson MS, et al. The validity of selfreported smoking: A review and meta-analysis. Am J Public Health. 1994;84(7):1086-1093.

16. Pokorski TL, Chen WW, Bertholf RL. Use of urine cotinine to validate smoking self-reports in US navy recruits. Addict Behav. 1994;19(4): 451-454.

17. Klesges RC, Debon M, Ray JW. Are self-reports of smoking rate biased? Evidence from the second national health and nutrition examination survey. J Clin Epidemiol. 1995;48(10):1225-1233.

18. Secker-Walker RH, Vacek PM, Flynn BS, Mead PB. Exhaled carbon monoxide and urinary cotinine as measures of smoking in pregnancy. Addict Behav. 1997;22(5):671-684.

19. Campbell E, Sanson-Fisher R, Walsh R. Smoking status in pregnant women: assessment of self-report against carbon monoxide (CO). Addict Behav. 2001;26(1):1-9.
20. Monninkhof E, Valk P van der, Palen $J$ van der, et al. The effect of a minimal contact smoking cessation programme in out-patients with chronic obstructive pulmonary disease: A pre-post-test study. Patient Educ Couns. 2004;52(3):231-236.

21. SRNT Subcommittee on Biochemical Verification. Biochemical verification of tobacco use and cessation. Nicotine Tob Res. 2002; $4(2): 149-159$

22. Jarvis MJ, Tunstall-Pedoe H, Feyerabend C, Vesey C, Saloojee Y. Comparison of tests used to distinguish smokers from nonsmokers. Am J Public Health. 1987;77(11):1435-1438.

23. Benowitz NL, Kuyt F, Jacob P, Jones RT, Osman AL. Cotinine disposition and effects. Clin Pharmacol Ther. 1983;34(5):604-611.

24. Murray RP, Connett JE, Lauger GG, Voelker HT. Error in smoking measures: Effects of intervention on relations of cotinine and carbon monoxide to self-reported smoking. Am J Public Health. 1993;83(9): 1251-1257.

25. Hilberink SR, Jacobs JE, Bottema BJAM, Vries H de, Grol RPTM. Smoking cessation in patients with COPD in daily general practice (SMOCC): Six months' results. Prev Med. 2005;41(5-6):822-827.

26. Hilberink SR, Jacobs JE, Schlösser M, Grol RPTM, de Vries H. Characteristics of patients with COPD in three motivational stages related to smoking cessation. Patient Educ Couns. 2006;61(3):449-457.

27. Hilberink SR, Jacobs JE, Breteler MHM, de Vries H, Grol RPTM. General practice counseling for patients with chronic obstructive pulmonary disease to quit smoking: Impact after 1 year of two complex interventions. Patient Educ Couns. 2010 Apr 27. [Epub ahead of print]

28. Prochaska JO, DiClemente CC. Stages and processes of self-change of smoking: Toward an integrative model of change. J Consult Clin Psychol. 1983;51(3):390-395.

29. Heatherton TF, Kozlowski LT, Frecker RC, Fagerström KO. The Fagerström test for nicotine dependence: A revision of the Fagerström Tolerance Questionnaire. Br J Addict. 1991;86(9):1119-1127.

30. Lende R van der, Orie NG. The MRC-ECCS questionnaire on respiratory symptoms (use in epidemiology). Scand J Respir Dis. 1972; 53(4):218-226.

31. Benowitz NL. Cotinine as a biomarker of environmental tobacco smoke exposure. Epidemiol Rev. 1996;18(2):188-204.

32. Bize R, Burnand B, Mueller Y, Rège Walther M, Cornuz J. Biomedical risk assessment as an aid for smoking cessation. Cochrane Database Syst Rev. 2009;2:CD004705.
International Journal of General Medicine

\section{Publish your work in this journal}

The International Journal of General Medicine is an international, peer-reviewed open-access journal that focuses on general and internal medicine, pathogenesis, epidemiology, diagnosis, monitoring and treatment protocols. The journal is characterized by the rapid reporting of reviews, original research and clinical studies across all disease areas.

\section{Dovepress}

A key focus is the elucidation of disease processes and management protocols resulting in improved outcomes for the patient.The manuscript management system is completely online and includes a very quick and fair peer-review system. Visit http://www.dovepress.com/ testimonials.php to read real quotes from published authors. 